\title{
Subunit Vaccine Based on Plant Expressed Recombinant Eimeria Gametocyte Antigen Gam82 Elicit Protective Immune Response against Chicken Coccidiosis
}

\author{
Sathish Kota ${ }^{1,2^{*}}$, Mohana Subramanian ${ }^{1,2,3}$, Bala Murugan Shanmugaraj ${ }^{2}$, Harini Challa ${ }^{2}$, Ponanna NM ${ }^{4}$, Srinivasan VA ${ }^{4}$ and Rajan Sriraman ${ }^{1,3}$ \\ ${ }^{1}$ Indian Immunologicals Itd, Hyderabad \\ ${ }^{2}$ Translational Research Platform for Veterinary Biologicals (TRPVB), Chennai \\ ${ }^{3}$ Biological. E, Hyderabad \\ ${ }^{4}$ National Dairy Development Board, Hyderabad
}

*Corresponding author: Dr. Sathish Kota, F1, Ramya Foundations, Bank Colony First Street, Chennai, Tel: +919603261720; E-mail: kotasathish17@gmail.com Received date: October 25, 2017; Accepted date: November 09, 2017; Published date: November 16, 2017

Copyright: $\odot 2017$ Sathish K, et al. This is an open-access article distributed under the terms of the Creative Commons Attribution License, which permits unrestricted use, distribution, and reproduction in any medium, provided the original author and source are credited.

\begin{abstract}
Avian coccidiosis is an intestinal tract infection caused by protozoan parasite of the genus Eimeria which is an economically important disease affecting poultry globally. In this study, E. maxima gametocyte antigen (Gam82) was expressed in tobacco leaves by agro-infiltration. The expression of Gam82 protein in plants was found to be 20 $\mathrm{mg} / \mathrm{kg}$ fresh weight. The recombinant protein was purified by affinity column chromatography and its immunogenicity was evaluated in chickens. The birds immunized with plant purified plant expressed Gam82 protein showed $39 \%$ of increased weight gain and $69 \%$ of reduced oocyst output compared to control birds indicating that the plant expressed Gam82 antigen can elicit protective immune response in immunized birds. Our study demonstrated that the plant expressed Gam82 antigen can potentially be a candidate subunit vaccine against coccidiosis.
\end{abstract}

Keywords: Agroinfiltration; Eimeria gametocyte antigen; Recombinant protein; Subunit vaccine

\section{Introduction}

Coccidiosis is one of the major devastating protozoan diseases of chicken with severe weight loss, reduction in feed conversion and increase in mortality. The disease results in severe economic loss for the poultry industry worldwide [1]. Although preventive medications and anti-coccidial drugs are available to control coccidiosis in chickens, development of drug resistant organism and drug residues in meat and egg are the major limitations of the current control method [2,3].

Development of cost-effective recombinant subunit vaccine may help to reduce the loss in poultry industry. Gametocyte and microneme proteins of genus Eimera are reported to be possible vaccine candidates to control coccidiosis $[4,5]$. The efficacy studies of $E$. coli expressed $E$. maxima gametocyte antigen and plant expressed $E$. tenella microneme-2 proteins was reported earlier [6,7]. Eimeria maxima gametocyte antigens appears to have great potential as part of a maternally applied vaccine against coccidiosis [8,9]. Purified recombinant protein from Eimeria maxima gametocytes (Gam82) has the ability to stimulate immunity against experimental infection with live parasites.

Currently commercial protein production relies mainly on E. coli, mammalian, yeast and insect cell expression systems. Plant expression platform has been an alternative to conventional protein expression systems due to its ease of transformation, low initial investment, high recombinant protein expression, easy scale up and the plant expressed proteins can undergo post-translational modifications [10,11]. Earlier reports had also showed that the recombinant antigens expressed in plants could be used as subunit vaccines [7,12-14]. Present study was aimed at evaluating the plant expressed recombinant $E$. maxima gametocyte antigen Gam82 against chicken coccidiosis using a bird challenge experiment.

\section{Materials and Methods}

\section{Chicken}

One-day-old commercial layer breed-BV 300 was obtained from Sri Venkateswara Hatcheries, Hyderabad, India. Chickens were fed and maintained in a pathogen (Eimeria) free environment and reared in clean brooder cages.

\section{Coccidial oocysts}

Wild type E. maxima oocysts used in the present study were isolated from an Eimeria infected farm in India. Oocysts were propagated in 3 weeks old birds by repeated passages [15]. Purity of oocyst suspension was assessed by species-specific nested-PCR for ribosomal Internal Transcribed Spacer I (ITS-I) region as described earlier [16].

\section{Cloning of Gams2 gene into plant expression vector}

E. maxima gametocyte antigen (Gam82) was amplified from a plasmid clone containing the full-length gene sequence of Gam82 [15] using Proof-start polymerase (Qiagen, USA). Gene specific primers flanked by BglII and NotI restriction sites (F-5'AAGCCAGATCTATGACGCGTGCGGCAGCGCTTG3' R-5'ATCTGCGGCCGCGTTGTATGTTTCCCATACAGT3') were used for the PCR amplification. The amplified products were ligated into plant expression vector pTRA-ERH and the plasmid clone was used to transform Agrobacterium cells (GV3101) by electroporation. 
Citation: Kota S, Subramanian M, Shanmugaraj BM, Challa H, Ponanna NM, et al. (2017) Subunit Vaccine Based on Plant Expressed Recombinant Eimeria Gametocyte Antigen Gam82 Elicit Protective Immune Response against Chicken Coccidiosis. J Vaccines Vaccin 8: 374. doi:10.4172/2157-7560.1000374

Page 2 of 6

\section{Agroinfiltration and RT-PCR}

The seeds of Nicotiana tabacum, cultivar Petit Havana SR1 were germinated in the greenhouse. 4 to 6 weeks old plants were used for Agroinfiltration [17]. Total RNA was extracted from the infiltrated tobacco leaves using RNAEasy plant-mini kit (Qiagen, USA), three days post infiltration and the amplification of specific mRNA was verified by RT-PCR using gene specific primers.

\section{SDS-PAGE analysis and immunoblotting}

The plant expressed recombinant Gam82 protein was purified by affinity chromatography and resolved on SDS-PAGE. The protein was further confirmed by Western Blot probed either with anti-HIS antibodies (Qiagen, Germany) or rabbit polyclonal sera reactive against Gam82 protein. The protein was also electro-blotted on to PVDF membrane (Hybond-P; GE-Healthcare, USA). The tobacco leaves extract were used as negative control, while $E$. coli expressed recombinant Gam82 protein was used as positive control.

\section{Immunization and efficacy study}

The in vivo immunogenicity of plant produced recombinant antigen was evaluated in Chickens. The study consisted of three treatment groups. Birds in Group I were immunized intramuscularly with $50 \mu \mathrm{g}$ of Gam82 protein with Freund's complete adjuvant whereas birds in Group II and III were used as control (PBS). Primary dose was followed by two booster doses on day 14 and 21. Number of birds in each group and treatment details are listed in Table 1.

\begin{tabular}{|l|l|l|l|l|}
\hline Group & $\begin{array}{l}\text { Immunogen } \\
(\boldsymbol{\mu} \text { g/bird) }\end{array}$ & $\begin{array}{l}\text { Immunization } \\
(\text { days })\end{array}$ & $\begin{array}{l}\text { Bleeding } \\
\text { (days) }\end{array}$ & Challenge \\
\hline $\begin{array}{l}\text { Group-I } \\
(\mathrm{N}=14)\end{array}$ & Gam82 (50) & $0,7,14,21$ & $\begin{array}{l}0,7,14,21, \\
28\end{array}$ & $\begin{array}{l}20,000 \text { oocyst/ } \\
\text { bird }\end{array}$ \\
\hline $\begin{array}{l}\text { Group-II } \\
(\mathrm{N}=14)\end{array}$ & PBS & $0,7,14,21$ & $\begin{array}{l}0,7,14,21, \\
28\end{array}$ & Unchallenged \\
\hline $\begin{array}{l}\text { Group-III } \\
(\mathrm{N}=14)\end{array}$ & PBS & $0,7,14,21$ & $\begin{array}{l}28 \\
28,14,21,\end{array}$ & $\begin{array}{l}20,000 \text { oocyst/ } \\
\text { bird }\end{array}$ \\
\hline
\end{tabular}

Table 1: Treatment groups and Immunization schedule

\section{Humoral immune response}

Blood was collected prior to each immunization and 7 days after final booster $\left(28^{\text {th }}\right.$ day post primary immunization). Serum antibody titers of the immunized proteins were evaluated using E. coli expressed recombinant Gam82 protein to assess specific antibody titers.

\section{Animal challenge experiment}

Nine days after second booster dose, treated and control groups were inoculated orally with 20,000 sporulated E. maxima oocysts. Birds were weighed prior to challenge and on 7 th and 11th days post challenge to determine the weight gain. The average weight gain of birds and oocyst shedding per gram of fecal matter was determined as described earlier [15]. An average of three counts per group was taken to enumerate oocyst shedding. The percent decrease in oocyst output compared to the mock-immunized but challenged birds was estimated [15].

\section{Results}

\section{Construction of recombinant plant expression vector and Agrobacterium transformation}

The Gam82 coding sequence $(1.8 \mathrm{~kb})$ was amplified from a plasmid clone by PCR and cloned into plant expression vector pTRA-ERH (Figure 1). Then the plasmid was transformed into Agrobacterium cells and the recombinant Agrobacterium clones were confirmed by PCR using gene specific primers.

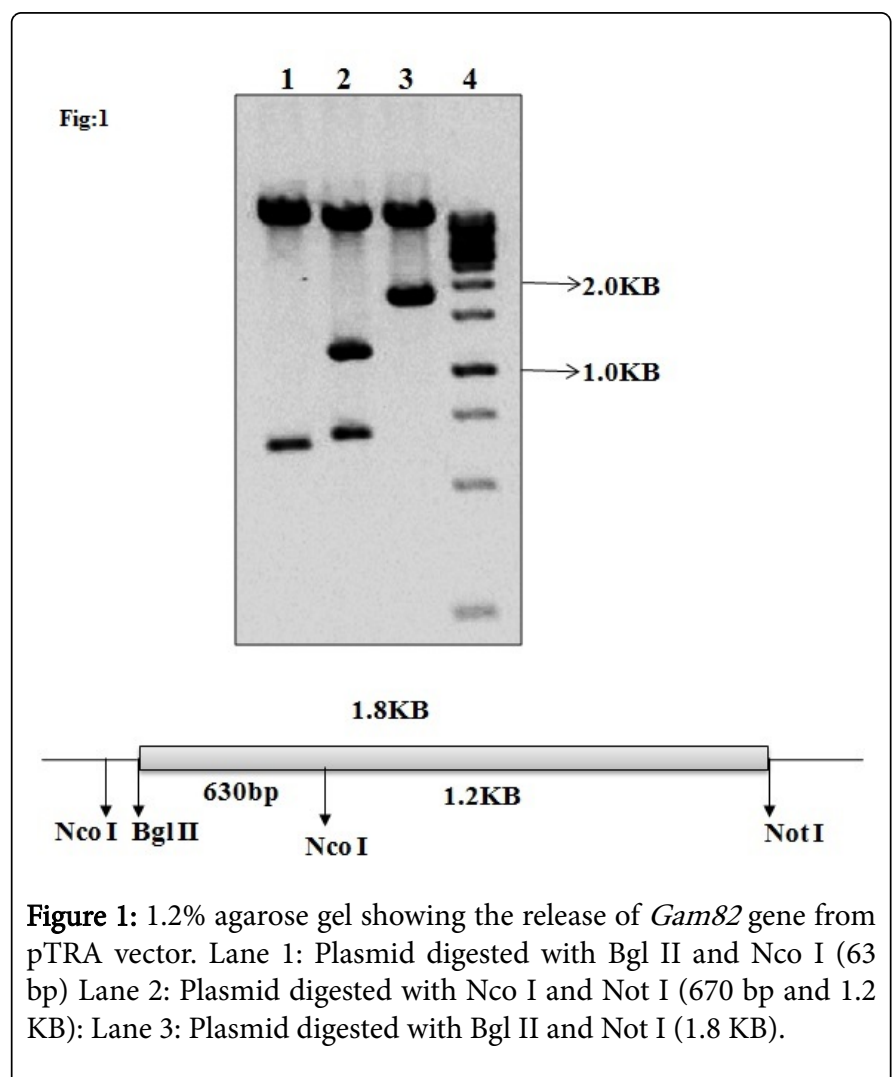

\section{Transient expression of Gam82 in N. tabacum}

The Agrobacterium harbouring pTRA-ERH vector was infiltrated into plants by vacuum infiltration. Three days post infiltration, the total RNA was extracted from the infiltrated leaves and used as a template for RT-PCR. In RT-PCR analysis, $1.8 \mathrm{~kb}$ amplicon was observed indicating the presence of mRNA in the infiltrated leaves (Figure 2). 
Citation: Kota S, Subramanian M, Shanmugaraj BM, Challa H, Ponanna NM, et al. (2017) Subunit Vaccine Based on Plant Expressed Recombinant Eimeria Gametocyte Antigen Gam82 Elicit Protective Immune Response against Chicken Coccidiosis. J Vaccines Vaccin 8: 374. doi:10.4172/2157-7560.1000374

Page 3 of 6

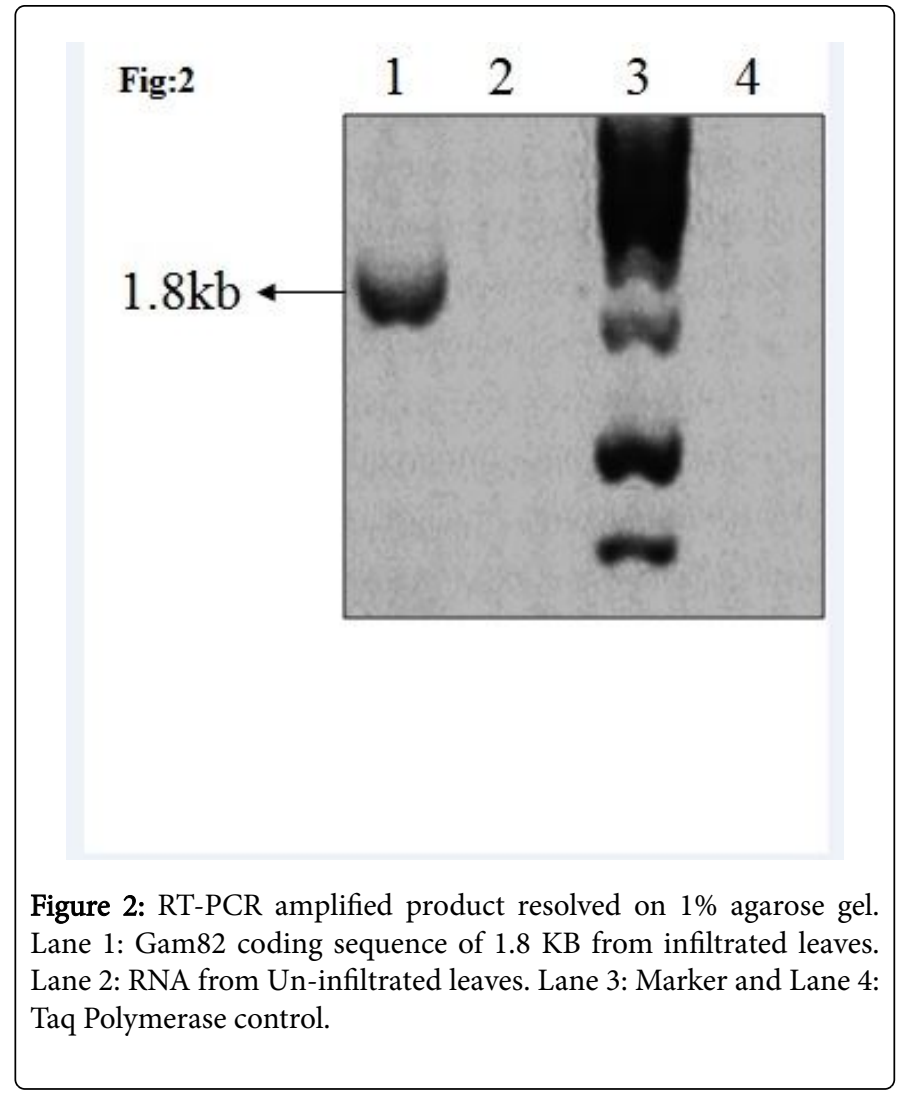

Upon confirmation of expression, the recombinant protein from the infiltrated leaves was purified by Nickel-affinity column chromatography. The purified proteins were resolved in SDS-PAGE and confirmed by western blotting probed either with rabbit polyclonal antibodies against Gam82 protein or anti-His antibody. A protein band of approximately $82 \mathrm{kDa}$ was observed in SDS-PAGE, corresponding to the size of Gam82 protein (Figures 3a and 3b).

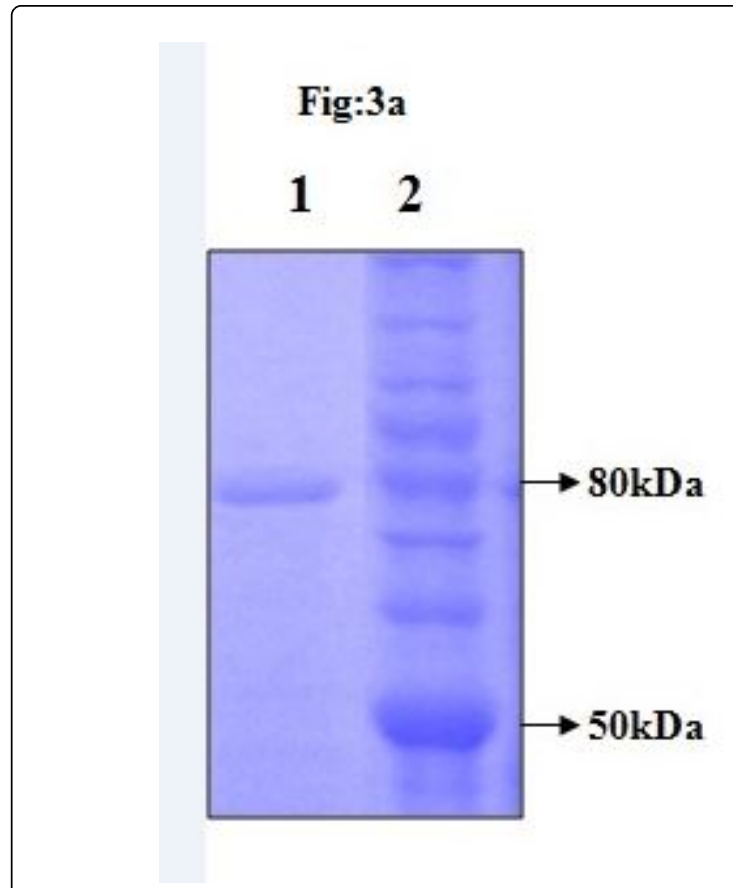

Figure 3a: PAGE showing plant expressed recombinant Gam82 protein stained with Coomasie blue.

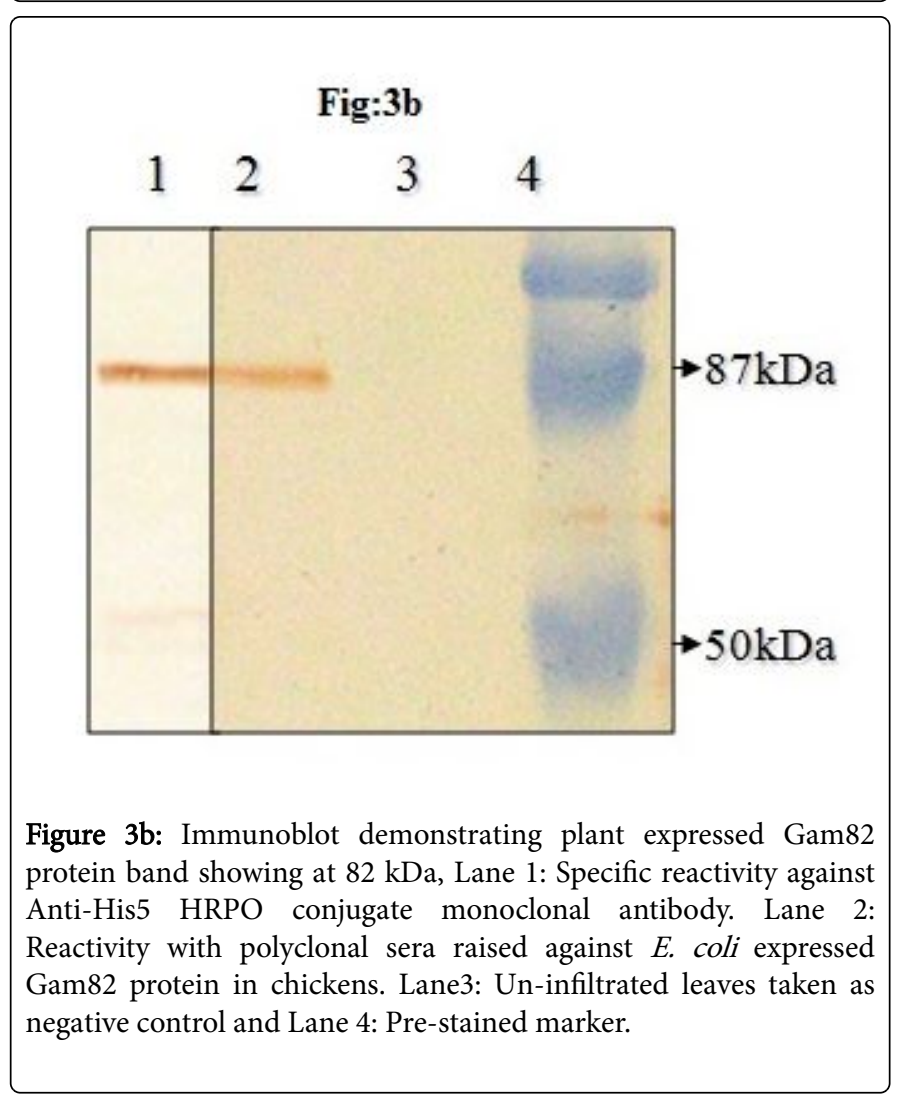


Citation: Kota S, Subramanian M, Shanmugaraj BM, Challa H, Ponanna NM, et al. (2017) Subunit Vaccine Based on Plant Expressed Recombinant Eimeria Gametocyte Antigen Gam82 Elicit Protective Immune Response against Chicken Coccidiosis. J Vaccines Vaccin 8: 374. doi:10.4172/2157-7560.1000374

Page 4 of 6

\section{Evaluation of humoral immune response}

The serum samples were collected from the birds on 14, 21 and 28 days post vaccination and the samples were analyzed in ELISA for the presence of Gam82 specific serum antibodies. The mean serum antibody titers in immunized birds were 133.3 ( \pm 50), $344.4( \pm 206.8)$ and $1333.3( \pm 800)$ on 14,21 and 28 DPV respectively (Figure 4).

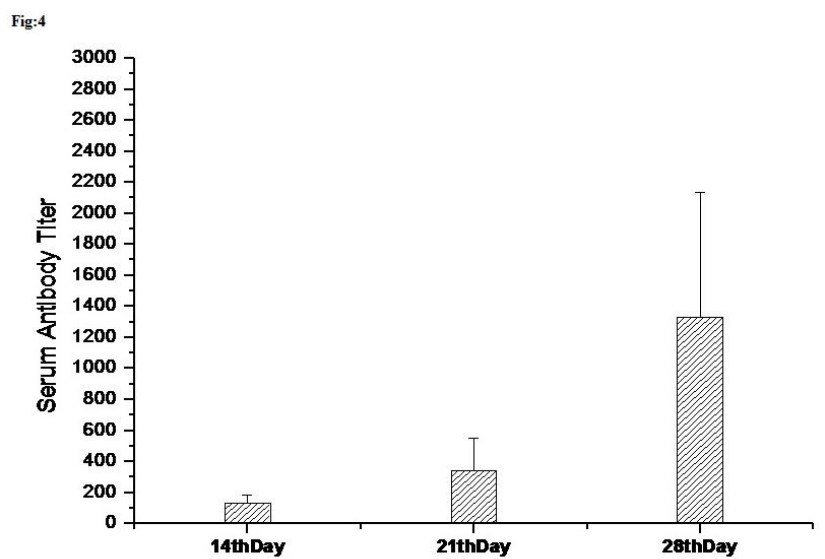

Figure 4: ELISA titers (mean \pm SD) of sera from birds immunized with plant expressed Gam82 protein. The assay was performed using indirect ELISA in a maxisorp plate coated with corresponding E. coli expressed protein. Antibody titers in the serum were determined as maximum sera dilution showing OD450 greater than mean $+3 \times \mathrm{SD}$ of pre-immune sera $(\mathrm{N}=14)$.

\section{Bird challenge experiments}

Weight gain: Birds in Groups I, and III were challenged with 20,000 virulent $E$. maxima oocysts. Weight gain was assessed on 7 and 11 days post-challenge $(\mathrm{dpc})$. The percentage increase in weight gain in the immunized birds compared to control group (Group II and III) was determined. It was observed that the birds immunized with plant purified Gam82 had 37.2\% ( \pm 52 ) and 20.6\% ( \pm 27 ) increase in weight gain on days 7 and 11 post-challenge. The difference in the mean weight gain was subjected to Student's t-test. The mean weight gain was significantly different in all immunized birds on $7 \mathrm{dpc}$ when compared with mock-immunized and challenged group $\left(\mathrm{p}<0.05^{\star} ; \mathrm{N}=14\right)$ (Figure 5).

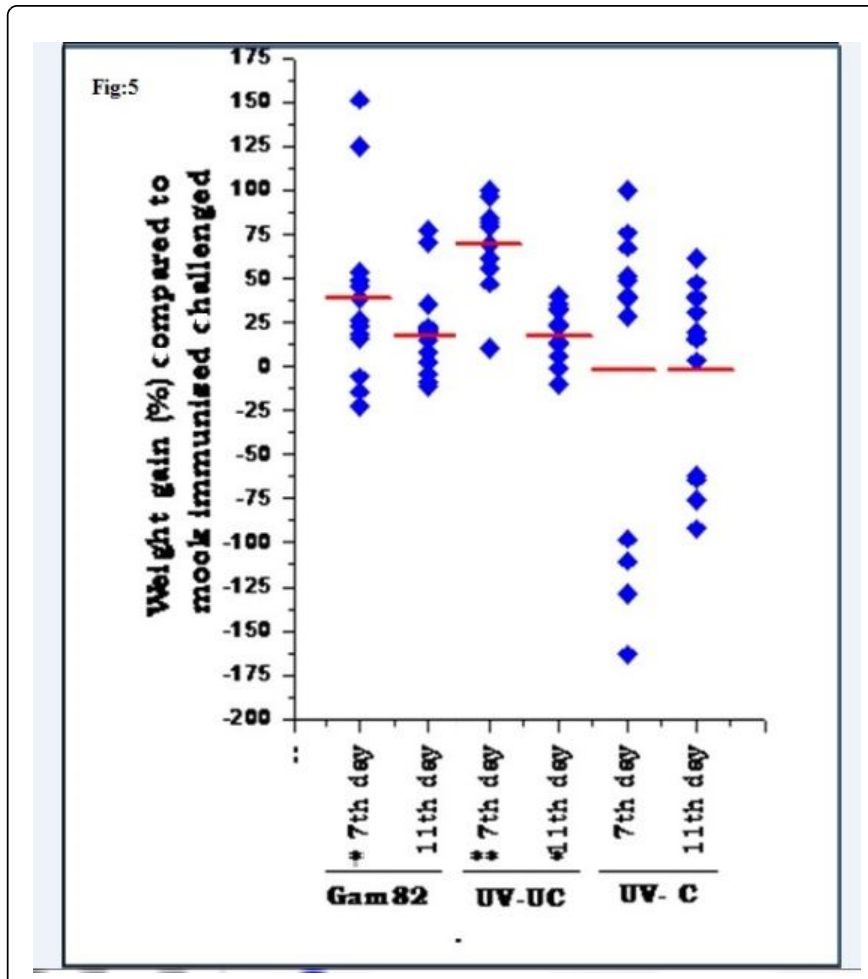

Figure 5: Percentage change in weight gain of immunized birds compared to mock immunized unchallenged Birds. Weight gain was calculated on 7th and 11th day after challenge. Mean value for the group is indicated by a line. The asterisks indicate significant change in weight gain compared to mock immunized and challenged birds $\left({ }^{*} \mathrm{p}<0.05 ;{ }^{* *} \mathrm{p}<0.01 ; \mathrm{N}=14\right)$.

Evaluation of oocyst output: Oocyst output of the immunized birds was compared with the oocyst output of control birds (Group II and III) after live Eimeria challenge. Immunization of birds with plant produced recombinant Gam82 reduced the oocyst output by $69 \%$. These results revealed that immunization of birds with the plant expressed recombinant gametocyte antigens imparted partial protection in chicken against parasite challenge (Figure 6). 
Citation: Kota S, Subramanian M, Shanmugaraj BM, Challa H, Ponanna NM, et al. (2017) Subunit Vaccine Based on Plant Expressed Recombinant Eimeria Gametocyte Antigen Gam82 Elicit Protective Immune Response against Chicken Coccidiosis. J Vaccines Vaccin 8: 374. doi:10.4172/2157-7560.1000374

Page 5 of 6

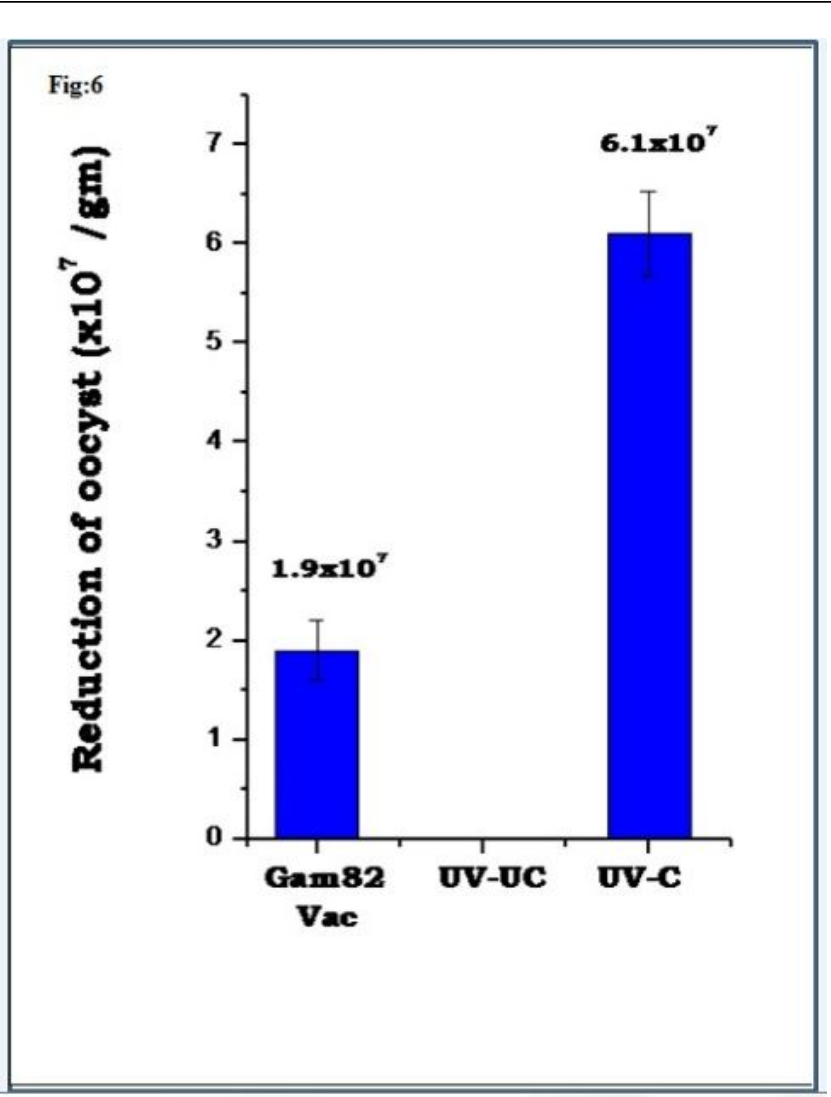

Figure 6: Oocyst output in immunized birds compared to mock immunized and challenged group. The oocyst shedding per gram of faces was determined using McMaster counting chamber. The bar represents an average of 3 counts per group.

\section{Discussion}

Coccidiosis is one of the major hindrances in the growth of poultry industry, as it affects the birds in both clinical and subclinical forms. Though medications are available to control the disease, the excessive usage of drugs may cause some potential side effects, such as drug residues in the product, emergence of resistant strains of Eimeria etc., which can be overcome by vaccination strategies. Recombinant subunit vaccines are used as a substitute to whole cell vaccines. However, large scale production of recombinant subunit proteins is essential to meet the global vaccination demand. Plant expression system is preferred as an attractive alternative for the large scale production of recombinant proteins in a short period at much reduced cost. Many vaccine candidates are expressed in plants and shown that plant purified recombinant proteins elicit potent immune response in animal models $[7,18-20]$.

Gam82 is $82 \mathrm{kDa}$ tyrosine-rich sexual stage glycoprotein of $E$. maxima, which is responsible for oocyst wall formation. Affinity purified gametocyte antigens including Gam82 from the sexual stage of $E$. maxima have been proved as potential vaccine targets in inducing transmission-blocking immunity [21]. Earlier studies reported that the gametocyte antigens are immunogenic and can be used as vaccine candidates to control coccidiosis $[21,22]$. The study conducted to evaluate the effects of in vivo vaccination with an $E$. maxima gametocyte recombinant protein, Gam82, on host immunity following live-challenge infection, promoted cell mediated immunity against experimental coccidiosis and reduced fecal oocyst shedding [23].

In the present study, we attempted to evaluate the in vivo immunogenicity of plant expressed Eimeria maxima gametocyte antigen Gam82. The Gam82 gene was cloned in pTRA-ERH expression vector and the protein was expressed in the leaves of $N$. tabacum by agro-infiltration. The recombinant protein was purified from the plant leaves by nickel affinity chromatography. The purified protein was confirmed in SDS-PAGE and western blot probed with anti-HIS antibody and anti-Gam 82 polyclonal sera. The yield of purified protein was found to be $20 \mathrm{mg} / \mathrm{kg}$ of leaves. Plant expressed recombinant Gam82 protein had induced high serum antibody response on 14, 21 and 28 days of post immunization in chickens when given as monovalent vaccine. Serum antibody response raised against plant expressed Gam82 protein was evaluated in ELISA using E. coli produced Gam82 as coating antigen. A challenge experiment was performed with live parasite in chickens after immunization. The average weight gain in vaccinated birds was higher both on 7 th day and 11th day post challenge, compared to unvaccinated birds (Figure 5 ). We observed that there was reduction of up to $69 \%$ of oocyst output from birds immunized with Gam82 protein (Figure 6). Our earlier work showed that the recombinant EtMIC1 and EtMIC2 protein expressed in plants elicit potent immune response which can be used as a subunit vaccine to control coccidiosis [7,24]. Earlier studies showed that the antibodies raised against gametocyte antigens play a major role in protection against homologous challenge [25,26]. The results obtained by immunizing E. coli produced recombinant Gam82 protein [23] are consistent with our results.

In summary, our study showed that plant produced recombinant gametocyte protein Gam82 was immunogenic and induced partial protection against live parasite challenge. This study explores the possibility of using plant produced recombinant Gam82 as a subunit vaccine against avian coccidiosis. As, coccidiosis is a complex disease that includes multiple stages of parasite invasion; inclusion of two or more recombinant subunit proteins as multivalent vaccine may help in complete protection against the disease.

\section{References}

1. Williams RB (1999) A compartmentalised model for the estimation of the cost of coccidiosis to the world's chicken production industry. Int J Parasitol 29: 1209-1229.

2. Dalloul RA, Lillehoj HS (2006) Poultry coccidiosis: recent progress on control measures and vaccination strategies. Expert Rev Vaccines 5: 143-163.

3. Chapman HD, Jeffers TK, Williams RB (2010) Forty years of monensin for the control of coccidiosis in poultry. Poult Sci 89: 1788-1801.

4. Lillehoj HS, Ding X, Quiroz MA, Bevensee E, Lillehoj EP (2005) Resistance to intestinal coccidiosis following DNA immunization with the cloned 3-1E Eimeria gene plus IL-2, IL-15, and IFN. Avian Dis 49: $112-117$

5. Lillehoj HS, Ding X, Dalloul RA, Sato T, Yasuda A (2005) Embryo vaccination against Eimeria tenella and E. acervulina infections using recombinant proteins and cytokine adjuvants. J Parasitol 91: 666-673.

6. Liu D, Cao L, Zhu Y, Deng C, Su S (2014) Cloning and characterization of an Eimeria necatrix gene encoding a gametocyte protein and associated with oocyst wall formation. Parasit Vectors 7: 27.

7. Sathish K, Sriraman R, Subramanian BM, Rao NH, Kasa B (2012) Plant expressed coccidial antigens as potential vaccine candidates in protecting chicken against coccidiosis. Vaccine 30: 4460-4464. 
Citation: Kota S, Subramanian M, Shanmugaraj BM, Challa H, Ponanna NM, et al. (2017) Subunit Vaccine Based on Plant Expressed Recombinant Eimeria Gametocyte Antigen Gam82 Elicit Protective Immune Response against Chicken Coccidiosis. J Vaccines Vaccin 8: 374. doi:10.4172/2157-7560.1000374

Page 6 of 6

8. Wallach M, Halabi A, Pillemer G, Sar-Shalom O, Mencher D (1992) Maternal immunization with gametocyte antigens as a means of providing protective immunity against Eimeria maxima in chickens. Infect Immun 60: 2036-2039.

9. Wallach M, Smith NC, Petracca M, Miller CM, Eckert J (1995) Eimeria maxima gametocyte antigens: potential use in a subunit maternal vaccine against coccidiosis in chickens. Vaccine 13: 347-354.

10. Ma JKC, Drake PMW, Christou P (2003) The production of recombinant pharmaceutical proteins in plants. Nature Rev Genet 4: 794-805.

11. Shanmugaraj BM, Ramalingam S (2014) Plant expression platform for the production of recombinant pharmaceutical proteins. Austin J Biotechnol Bioeng 1: 6 .

12. Meyers A, Chakauya E, Shephard E, Tanzer FL, Maclean J (2008) Expression of HIV-1 antigens in plants as potential subunit vaccines. BMC Biotechnol 8: 53.

13. Bhoo SH, Lai H, Ma J, Arntzen CJ, Chen Q, et al. (2011) Expression of an immunogenic Ebola immune complex in Nicotiana benthamiana. Plant Biotechnol J 9: 807-816.

14. Sathish K, Sriraman R, Subramanian BM, Rao NH, Kasa B (2012) Plant expressed coccidial antigens as potential vaccine candidates in protecting chicken against coccidiosis. Vaccine 30: 4460-4464.

15. Ruiz V, Mozgovoj MV, Santos MJD, Wigdorovitz A (2015) Plantproduced viral bovine vaccines: what happened during the last 10 years? Plant Biotechnol J 1: 1-7.

16. Subramanian BM, Sriraman R, Hanumantha Rao N, Raghul J, Thiagarajan D (2008) Cloning, expression and evaluation of the efficacy of a recombinant Eimeria tenella sporozoite antigen in birds. Vaccine 26: 3489-3496.

17. Bhaskaran MS, Venkatesan L, Aadimoolam R, Tirunelveli JH, Sriraman R (2010) Sequence diversity of internal transcribed spacer-1 (ITS-1) region of Eimeria infecting chicken and its relevance in species identification from Indian field samples. Parasitol Res 106: 513-521.
18. Kapila J, Rycke RD, Van Montagu M, Agenon G (1997) An Agrobacterium-mediated transient gene expression system for intact leaves. Plant Sci 122: 101-108.

19. McGarvey PB, Hammond J, Dienelt MM, Hooper DC, Fu ZF (1995) Expression of the rabies virus glycoprotein in transgenic tomatoes. Biotechnology 13: 1484-1487.

20. Khandelwal A, Sita GL, Shaila MS (2003) Expression of hemagglutinin protein of rinderpest virus in transgenic tobacco and immunogenicity of plant-derived protein in a mouse model. Virology 308: 207-215.

21. Thanavala Y, Mahoney M, Pal S, Scott A, Richter L (2005) Immunogenicity in humans of an edible vaccine for hepatitis B. Proc Natl Acad Sci USA 102: 3378-3382.

22. Wallach M, Pillemer G, Yarus S, Halabi A, Pugatsch T (1990) Passive immunization of chickens against Eimeria maxima infection with a monoclonal antibody developed against a gametocyte antigen. Infect Immun 58: 557-562.

23. Pugatsch T, Mencher D, Wallach M (1989) Eimeria maxima: isolation of gametocytes and their immunogenicity in mice, rabbits, and chickens. Exp Parasitol 68: 127-134.

24. Jang SI, Lillehoj HS, Lee SH, Lee KW, Park MS, et al. (2010) Eimeria maxima recombinant Gam82 gametocyte antigen vaccine protects against coccidiosis and augments humoral and cell-mediated immunity. Vaccine 28: 2980-2985.

25. Sathish K, Sriraman R, Subramanian BM, Rao NH, Balaji K (2011) Plant expressed EtMIC2 is an effective immunogen in conferring protection against chicken coccidiosis. Vaccine 29: 9201-9208.

26. Wallach MG, Mencher D, Yarus S, Pillemer G, Halabi A (1989) Eimeria maxima: identification of gametocyte protein antigens. Exp Parasitol 68: 49-56. 